\title{
Modelling and Performance Evaluation of a Translucent OBS Network Architecture
}

\author{
Oscar Pedrola*, Davide Careglio*, Miroslaw Klinkowski ${ }^{\dagger}$, and Josep Solé Pareta* \\ * CCABA, Universitat Politécnica de Catalunya (UPC), Barcelona, Spain, e-mail: opedrola@ac.upc.edu \\ $\dagger$ National Institute of Telecommunications (NIT), Warsaw, Poland, e-mail: mklinkow@itl.waw.pl
}

\begin{abstract}
Most research works in optical burst switching (OBS) networks do not take into account the impact of physical layer impairments (PLIs) either by considering fully transparent (i.e., using optical $3 R$ regeneration) or opaque (i.e., electrical $3 R$ regeneration) networks. However, both solutions are not feasible for different reasons. In this paper, we propose a novel translucent OBS network architecture which aims at bridging the gap between the transparent and opaque solutions. In order to evaluate its performance, two different joint regenerator placement and routing heuristics are provided. Simulation results show that our translucent network model achieves performance results as good as those obtained with an opaque solution but with considerably less regenerators.
\end{abstract}

\section{INTRODUCTION}

Although recent advances in optical technologies are fostering the deployment of fully transparent (i.e., all-optical switching) optical transport networks (OTNs), the physical layer impairments (PLIs) of the optical domain prevent it from taking place, at least, in the short-medium term [1]. For that very reason, translucent OTNs are a promising solution for bridging the gap between opaque (i.e., optical-electrical-optical (O/E/O) conversion at each node) and transparent networks. Indeed, translucent networks combine features of both opaque and transparent networks allowing signal regeneration only at selected points in the network [2].

Among the WDM solutions proposed, optical burst switching (OBS) has appeared as a potential candidate for nextgeneration OTNs. In short, OBS overcomes the technological constraints of optical packet switching (OPS) and the bandwidth inefficiency of optical circuit switching (OCS). In this paper, we propose a novel translucent OBS network architecture and evaluate its performance through two distinct heuristics for the placement of sparse $\mathrm{O} / \mathrm{E} / \mathrm{O}$ regenerators in the network. The study here presented follows an off-line approach since both the routing and regenerator placement (RRP) decisions are taken during the network planning stage. The consideration of a dynamic traffic matrix, by contrast, would result in the evaluation of an on-line problem, and thus, is left out of the scope of this paper.

The rest of the paper is organized as follows. In Section II, we survey the previous work in this topic and highlight the main contributions of this paper. In Section III, we give a complete description of the proposed translucent OBS network architecture. In Section IV, a detailed formulation of the heuristics that solve the RRP problem are given. In Section V, we conduct a series of extensive simulation experiments to compare and evaluate the performance of our proposals. Finally, in Section VI we draw some conclusions and indicate future works.

\section{RELATED WORK AND CONTRIBUTIONS}

The evolution of optical networks from traditional opaque towards transparent network architectures has brought to light the serious impact that PLIs have on the optical end-to-end signal quality. In fact, due to these physical constraints and the lack of optical regeneration, a fully transparent longhaul network solution is still not viable. Therefore, the study and evaluation of translucent OCS networks has recently received increasing attention from the research community (see e.g., [3]-[5]).

This is not the case for OBS where the vast majority of the works consider that either an ideal physical layer or optical regenerators at every channel, port and switching node of the network are available (i.e., fully transparent OBS). Recently, however, owing to the increasing interest on assessing the effect of the PLIs in the optical networks field, we find few interesting works that involve the PLI constraint in the evaluation of the OBS network performance. For example, some impairment-aware scheduling policies with the aim of minimizing the burst loss probability are presented in [6]. Another interesting study that incorporates PLIs in the definition of an algorithm for distributing manycasting services over an OBS network can be found in [7]. An extensive study that evaluates the design and maximum size and throughput for OBS core nodes considering the effects of a range of PLIs such as amplifier noise, crosstalk of WDM channels, gain saturation and dynamics can be found in [8]. However, in this work, all nodes are equipped with $\mathrm{O} / \mathrm{E} / \mathrm{O}$ wavelength converters (which also perform electrical regeneration), and thus, an opaque OBS network is being considered.

Because of the high cost of the O/E/O devices, the minimization of the number of such components present in the network is crucial to the problem's success. Hence, the socalled regenerator placement (RP) problem [3] arises. An RP algorithm must be able to find both the minimum number of regenerators and their exact location for a network topology so that for every source-destination node pair a path can be established. However, RP is an NP-complete problem [4] and heuristic methods are generally employed. Recent studies in OCS (e.g. [5]) show that better network performance can be 
achieved by treating the RP and the routing problem together in the so-called RRP problem.

In this work we propose a novel translucent OBS architecture that copes with all the requirements of such networks. In fact, none of the studies in the literature considering PLIs in OBS networks tackle the issue of designing a complete translucent OBS network. In this work, we first model and evaluate a feasible (i.e., with commercially available or at most lab trial devices) all-optical OBS network which has $\mathrm{O} / \mathrm{E} / \mathrm{O}$ regenerators available at selected nodes; secondly, we propose and evaluate two distinct RRP heuristics which take into account the optical signal to noise ratio (OSNR) at the receiving end as PLI constraint.

\section{Translucent OBS Network Model}

In this section, we present in detail our proposed translucent OBS network architecture. First, we provide the model for an all-optical OBS core node which incorporates a limited number of shared electrical regenerators. Second, we show the model that we consider for the calculation of the OSNR level. Finally, a power budget and noise analysis of the signal path between two OBS switching nodes is provided.

\section{A. Translucent Node Architecture}

The node architecture here presented is based on the model proposed in [8], where an opaque OBS network solution is considered. To be precise, the authors present two semiconductor optical amplifier (SOA)-based node architectures for OBS networks, namely broadcast-and-select (BAS) and tune-andselect (TAS). Both architectures rely on the promising SOA technology and on wavelength converters performing electrical $3 \mathrm{R}$ regeneration as their fundamental switch modules. Indeed, SOA as switching elements (SW-SOA) bring some interesting advantages such as high on/off ratios and loss compensation capabilities. Among them, the authors conclude that TAS is more appropriated for OBS networks because BAS displays some major drawbacks (e.g., high power requirements and large inter channel crosstalk) inherent to its architecture.

In this paper, we modify the aforementioned opaque TAS OBS core node architecture by replacing each inline electrical wavelength converter with a block consisting of a tunable laser and a wavelength conversion-type SOA (WC-SOA) device. Hence, this modified TAS node architecture (depicted in Fig. 1) is able to perform an all-optical switching operation. The node consists of $N$ input/output fibers with $M$ channels each and a limited number $R$ of regenerators available. After the signal is amplified by the erbium-doped fiber amplifier (EDFA) pre-amplifier at each node input port, it is demultiplexed and passes through a fixed-input and variable-output WC-SOA. Then, the signal is split into $N+1$ branches, one per each fiber plus an extra branch that allows the access to the regenerator pool, which consists of a set of $R$ fixed receivers, an electrical buffering stage and a set of $R$ lasers emitting in predefined wavelengths (i.e., $\lambda_{1}, \ldots, \lambda_{R}$ ). The signal is then transported to the output ports of the node following the decisions of the OBS node controller by turning the SW-SOAs

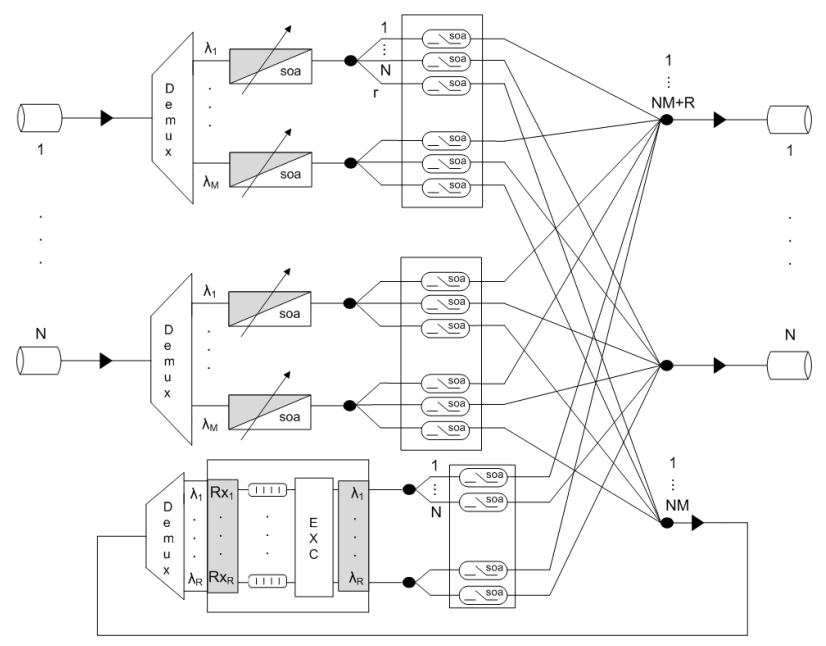

Fig. 1. Translucent TAS OBS core node architecture.

either ON or OFF. After the combiner stage, an EDFA booster amplifier provides the signal with enough power to cope with the losses of the first fiber span. Note also that, in this case, the combiners behind the SW-SOAs port merge $N M+R$ signals at each output port as a consequence of the presence of the regenerator pool.

It is worth mentioning that since the output of the WC-SOA is handled by the OBS node controller, all wavelengths from all input ports have the same privileges when requesting a regenerator, and thus, fairness in the access to the regenerator pool is provided by this architecture.

\section{B. OSNR network model}

In this OSNR model, the impact of PLIs is captured by considering the power of both the signal and the noise, which are affected by different gains and losses along the path, at the destination node. Although there exist many other PLIs, either linear or non-linear, here we consider the amplified spontaneous emission (ASE) noise introduced by both the EDFA and SOA amplifiers as the significant signal impairment factor. In fact, ASE is commonly considered as the most severe impairment that limits the reach and capacity of optical systems. In this case, OSNR is defined as the ratio between the signal channel power and the power of the ASE noise in a specified bandwidth (e.g., $0.1 \mathrm{~nm}$ are usually taken by convention) and is generally the fundamental metric which literature studies are based on. For instance, an OSNR model and its evaluation in transparent OCS networks is proposed in [9], while in [10] such model is experimentally validated in translucent OCS networks.

To quantify the OSNR degradation along the optical path, we define the optical path OSNR $\left(P_{\text {osnr }}\right)$ by taking advantage of the model described in [11]. Specifically, the OSNR consists of two main components, namely the link and node OSNR that we denote as $L_{o s n r}$ and $N_{o s n r}$ respectively. Since a link is composed of several amplifier spans, each ending with an inline EDFA amplifier, the longer the path the higher the impact of the ASE noise in the OSNR received. Similarly, to minimize 


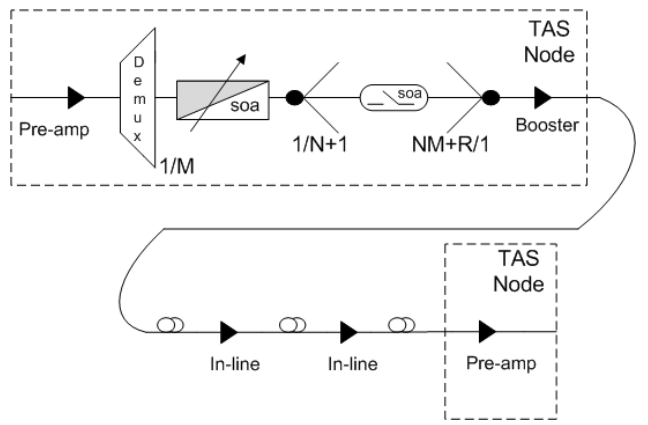

Fig. 2. Signal path between two TAS OBS core nodes.

the ASE effect caused by the internal node amplifiers, gain values should be designed such that each node presents an OSNR level as high as possible. We can compute $P_{\text {osnr }}$ for an optical end-to-end path traversing $k$ links by using the following equation,

$$
P_{\text {osnr }}=1 /\left(\sum_{i=1}^{k} \frac{1}{L_{\text {osnr }}^{i}}+\sum_{i=1}^{k} \frac{1}{N_{\text {osnr }}^{i}}\right),
$$

where for a link consisting of $r$ amplifier spans, $L_{\text {osnr }}^{i}$ is defined as follows,

$$
L_{o s n r}^{i}=1 /\left(\sum_{j=1}^{r} \frac{1}{A S_{o s n r}^{j}}\right)
$$

where $A S_{\text {osnr }}^{j}$ is the amplifier span OSNR, which can be calculated as,

$$
A S_{\text {osnr }}^{j}[d B]=P_{j}[d B m]-Q N[d B m]-F_{j}[d B]-G_{j}[d B],
$$

where $P_{j}, Q N, F_{j}, G_{j}$, correspond to the output power after the $j^{\text {th }}$ amplifier span, the quantum noise, the noise figure and the gain of the $j^{t h}$ amplifier (i.e., either EDFA in-line or pre-amplifier) respectively. The expression that we use to compute $N_{\text {osnr }}$ is equal to the one that we have defined for $A S_{\text {osnr }}$, however, due to the presence of several components (e.g., amplifiers, splitters and combiners) in our translucent node, both an equivalent noise and gain figure, namely $F_{e q}$ and $G_{e q}$ respectively, have to be derived.

In the next subsection, we provide specific values for all these figures by considering performance parameter values obtained from datasheets of commercially available devices (see e.g., [12]-[13]).

\section{Power Budget and Noise Analysis}

We consider the power and noise constraints together in order to evaluate the OSNR of a signal that follows the characteristic path between two TAS neighboring nodes depicted in Fig. 2. Component specifications are provided in Table I and the power constraints for this analysis are: the output power of the node (i.e., output of the EDFA booster amplifier) set to

\begin{tabular}{|c|c|c|c|c|}
\hline \multicolumn{5}{|c|}{ Span length $=65 \mathrm{~km}$} \\
\hline \multicolumn{5}{|c|}{ Fiber attenuation $=0.2 d B / \mathrm{km}+3 d B$ (cable margin) } \\
\hline \multicolumn{5}{|c|}{ Quantum Noise $=-58 d B m$} \\
\hline EDFA & booster & \multicolumn{2}{|c|}{ in-line } & pre-amp \\
\hline noise figure & $5.5 d B$ & \multicolumn{2}{|c|}{$5.5 d B$} & $5.5 d B$ \\
\hline max. output power & $18 d B m$ & \multicolumn{2}{|c|}{$18 d B m$} & $13 d B m$ \\
\hline min. output power & $-15 \mathrm{dBm}$ & \multicolumn{2}{|c|}{$-25 d B m$} & $-30 d B m$ \\
\hline max. gain & $15 d B$ & \multicolumn{2}{|c|}{$25 d B$} & $20 d B$ \\
\hline \multicolumn{2}{|l|}{ SOA } & WC-SOA & \multicolumn{2}{|c|}{ SW-SOA } \\
\hline \multicolumn{2}{|c|}{ noise figure } & $9 d B$ & \multicolumn{2}{|c|}{$10 d B$} \\
\hline \multicolumn{2}{|c|}{ max. output power } & $5 d B m$ & \multicolumn{2}{|c|}{$3 d B m$} \\
\hline \multicolumn{2}{|c|}{ min. output power } & $-25 d B m$ & \multicolumn{2}{|c|}{$-25 d B m$} \\
\hline max. ga & & $16 d B$ & \multicolumn{2}{|c|}{$10 d B$} \\
\hline \multicolumn{2}{|c|}{ rise-fall time } & - & \multicolumn{2}{|c|}{$500 p s$} \\
\hline \multicolumn{3}{|c|}{ WDM Demux insertion loss } & \multicolumn{2}{|c|}{$5.5 d B$} \\
\hline \multicolumn{3}{|c|}{ Splitter insertion loss } & \multicolumn{2}{|c|}{$0.5-1) d B$} \\
\hline \multicolumn{3}{|c|}{ Combiner insertion loss } & \multicolumn{2}{|c|}{$(1.5-2) d B$} \\
\hline
\end{tabular}
$0 \mathrm{dBm} / \mathrm{channel}$, and its input power (i.e., input of the EDFA pre-amplifier) set by link losses to $-16 \mathrm{dBm} /$ channel.
TABLE I

PARAMETER VALUES CONSIDERED

From (3) and bearing in mind that the objective is to have a $N_{o s n r}$ as high as possible, it can be inferred that both $F_{e q}$ and $G_{e q}$ must be designed so that its resultant value is minimized. For this particular case, the equivalent noise and gain figures of the TAS node are obtained as follows,

$$
\begin{gathered}
F_{e q}=F_{w c-s o a}+\frac{M F_{\text {sw-soa }}-1}{\frac{G_{w c-\text { soa }}}{L_{\text {splitter }}}}+\frac{F_{\text {edfa-booster }}-1}{\frac{G_{w c-\text { soa }} G_{\text {sw-soa }}}{L_{\text {splitter }} L_{\text {combiner }}}}, \\
G_{e q}=\frac{G_{w c-\text { soa }} G_{\text {sw-soa }} G_{\text {edfa-booster }}}{L_{\text {splitter }} L_{\text {combiner }}} .
\end{gathered}
$$

The most critical point is the combiner where, in the worst case, the ASE noise power from $M$ SW-SOAs is merged. Both the pre-amplifier and booster EDFAs and the WC-SOA and SW-SOA have to be used to compensate the internal losses. Their gain values must be carefully designed so that both equivalent figures are minimized and the power constraints are respected. In order to minimize $F_{\text {eq }}$, it can be deduced from (4) that, as long as the saturation output power is not reached, it is better to set the gain on the WC-SOA. In this way, the impact of the $M$ ASE powers is reduced. The EDFAs pre-amplifier and booster and SW-SOA gains, by contrast, are kept as low as allowed by the system power requirements. The exact set up for each component depends on the number of input/output ports of each particular node, which eventually define the splitting losses that are to be covered by $G_{e q}$.

In Fig. 3, we show the result of the application of the OSNR model presented throughout this whole section considering the optical end-to-end paths of two Pan-European networks, namely Core and Large network topologies (see Section V for the simulation details). Figure 3 makes it clear that the length, and thus, the number of amplifier spans, have a strong impact on the received OSNR. Consequently, all bursts arriving at the destination node with an accumulated OSNR value under the threshold can not be read correctly, and thus, are discarded.

\section{Routing And Regenerator Placement}

In this Section we focus on an RRP problem in a translucent OBS network. Specifically, we begin by presenting the design 


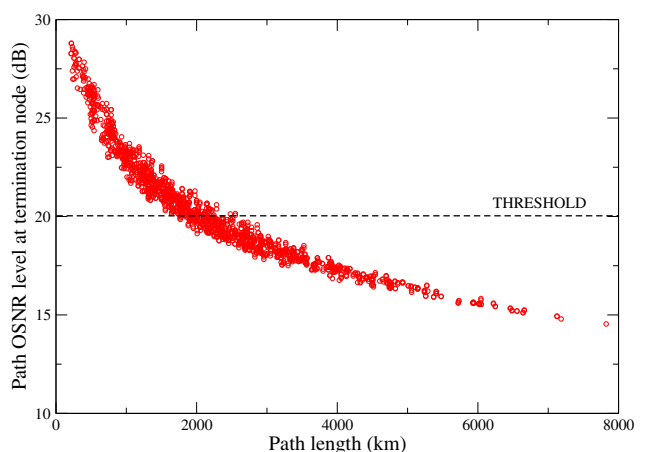

Fig. 3. Pan-European paths OSNR evaluation.

assumptions and by introducing the corresponding notation. Then, we propose some heuristics to solve the RRP problem, i.e. found the explicit paths to be used to route bursts through the network, the placement of regenerators in selected nodes on those paths, and the dimensioning of regenerators.

At this point, it is worth pointing out that since we are addressing an off-line strategy, we can assume that burst control packets (BCPs) are provided at their respective source node with the information on the set of nodes where their corresponding data burst will be regenerated.

\section{A. Notation}

We use $\mathcal{G}=(\mathcal{V}, \mathcal{E})$ to denote the graph of an OBS network; the set of nodes is denoted as $\mathcal{V}$, and the set of unidirectional links is denoted as $\mathcal{E}$. Let $d_{e}$ denote the length of link $e$.

Let $\mathcal{P}$ denote the set of predefined candidate paths between source $s$ and termination $t$ nodes, $s, t \in \mathcal{V}$, and $s \neq t$. Each path $p \in \mathcal{P}$ is identified with a subset $p \subseteq \mathcal{E}$. Let $\delta_{p}=$ $\sum_{e \in p} d_{e}$ be the length of path $p$. Let $s_{p}$ and $t_{p}$ denote the source and termination nodes of $p$. Let $\mathcal{V}_{p}$ denote the set of intermediate nodes, that is, excluding $s_{p}$ and $t_{p}$, on path $p$.

Let $\mathcal{D}$ denote the set of demands, where each demand corresponds to a pair of source-termination nodes. For each demand $d \in \mathcal{D}, h_{d} \in \mathbb{R}_{+}$denotes the volume of burst traffic.

We assume the network operates with explicit source routing. Let $\mathcal{P}_{d} \subseteq \mathcal{P}$ denote the set of candidate paths supporting demand $d ; \mathcal{P}=\bigcup_{d \in D} \mathcal{P}_{d}$. Each subset $\mathcal{P}_{d}$ comprises a (small) number of paths, for example, $k$ shortest paths. We consider single-path routing and, accordingly, only one path $p_{d} \in \mathcal{P}_{d}$ is selected as the valid path to be followed by all bursts belonging to demand $d$. Let $\mathcal{Q}$ denote the set of valid paths, $\mathcal{Q}=\left\{p_{d}, d \in \mathcal{D}\right\}$.

Let $\mathcal{R}_{d}$ denote the set of nodes where the regeneration is performed on valid path $p_{d}$. Let $r_{v}$ indicate the number of paths requiring regeneration in node $v \in \mathcal{V}$; note that the value of $r_{v}$ is subject to changes during the algorithm procedure.

\section{B. The RRP framework}

To solve the RRP problem we propose the following algorithm, which is decomposed into three main phases.

The first two phases are the routing and the regenerator placement. They are performed one after the other and iteratively, for each demand $d \in \mathcal{D}$. The result of this step is the set of valid routing paths $\mathcal{Q}$ and, for each $d \in \mathcal{D}$, the set of nodes $\mathcal{R}_{d}$ in which the regeneration of an optical burst, when sent on path $p_{d}$, has to be performed. Although the order of the iteratively processed demands may result in different solutions, still we observed that the algorithm performance does not vary significantly. Thus, we consider an arbitrary order.

The last phase is the regenerator dimensioning. Having found valid paths and regeneration nodes, this step determines the number of regenerators to be installed in these nodes.

Below we present the details of the algorithm subroutines.

\section{Routing phase}

In the routing phase the algorithm makes a decision on the selection of (single) path $p_{d}$ from the set of candidate paths $\mathcal{P}_{d}$. We propose two alternative methods, both described below.

\section{i) Regenerator Grouping-oriented $(R G)$ selection}

The RG method aims at the selection of paths that tends to group the regenerators in nodes as much as possible. First, it checks if there is a path $p \in \mathcal{P}_{d}$ such that the OSNR requirements are met; if yes, it selects the shortest one. Otherwise, the algorithm performs the search for the set of paths $\mathcal{P}_{d}^{*}$ with the maximal number of regenerators placed so far, that is, $\mathcal{P}_{d}^{*}=\left\{p: \sum_{v \in \mathcal{V}_{p}} r_{v} \geq \sum_{v \in \mathcal{V}_{q}} r_{v}, p, q \in \mathcal{P}_{d}\right\}$ and, among those paths, the (arbitrary) selection of the shortest one.

\section{ii) Link Congestion Reduction-oriented (LCR) selection}

The objective of the LCR method is to select paths that lead to the congestion reduction in network links. To achieve it we make use of the Linear Programming (LP)-based multipath routing algorithm presented in Section 4.2 in [14]. To find a single path $p_{d}$ for each demand $d$, we modify the LP formulation by forcing routing variables to be binary and then solve the resulting Mixed Integer Linear Programming (MILP) problem. Note that since we find a solution for all $d \in \mathcal{D}$ at once, it is enough to run the LCR procedure only once (e.g., at the beginning of the RRP algorithm).

These methods result in two algorithms, namely, the RG algorithm and the LCR algorithm.

\section{Regenerator placement phase}

In this phase, the search for appropriate location of regenerators in intermediate nodes on path $p_{d}$ is performed. Note that this step is run only if necessary (i.e., whenever $p_{d}$ does not meet the OSNR requirements). Let $\Omega_{d}$ be the set of subpaths of $p_{d}$ to be processed; we begin with $\Omega_{d}=\left\{p_{d}\right\}$.

First, the algorithm checks if among intermediate nodes $\mathcal{V}_{p}$ there are nodes with regenerators already located (for previously processed paths). If so, among those nodes it selects node $v$, the nearest one to the middle of the path (with respect to the number of hops), and considers it as a regenerative node for path $p_{d}$, i.e., $\mathcal{R}_{d}=\mathcal{R}_{d} \cup\{v\}$. Path $p_{d}$ can be decomposed into two subpaths, namely, $p_{s-v}$ (from source to $v$ ) and $p_{v-t}$ (from $v$ to termination). Accordingly, $\Omega_{d}=\Omega_{d} \cup\left\{p_{s-v}, p_{v-t}\right\} \backslash\left\{p_{d}\right\}$. Now, perform the following procedure: 
1) If $\Omega_{d}$ is empty, terminate the procedure, otherwise, take subpath $q \in \Omega_{d}$.

2) If $q$ meets the OSNR requirements, remove it from $\Omega_{d}$ and repeat step 1 .

3) Let $q^{*}$ be a clone of $q$.

4) Remove the last link (and node) from $q^{*}$. If $q^{*}$ does not meet the OSNR requirements, repeat step 3.

5) Consider $t_{q^{*}}$ as the regenerative node, $\mathcal{R}_{d}=\mathcal{R}_{d} \cup\left\{t_{q^{*}}\right\}$.

6) Add subpath $q \backslash q^{*}$ to $\Omega_{d}$ and repeat step 1 .

Eventually, in the regenerator placement phase, whenever node $v \in \mathcal{V}$ is added to set $\mathcal{R}_{d}$, the indicator $r_{v}$ is incremented.

\section{E. Regenerator dimensioning phase}

Let $\mathcal{R}=\bigcup_{d \in \mathcal{D}} \mathcal{R}_{d}$ be the set of all nodes where the regenerators have to be installed. We take the assumption that bursts entering node $v \in \mathcal{R}$ and requiring regeneration compete in the access to regeneration resources. The load of such burst traffic is (approximately) given by $\rho_{v}=\sum_{d \in \mathcal{D}, v \in \mathcal{R}_{d}} h_{d}$. In order to determine the number of regenerators required in node $v$ we define a dimensioning function $f\left(\rho_{v}, B\right):\left(R^{+}, R^{+}\right) \mapsto \mathbb{Z}^{+}$, where $B$ represents some target burst blocking probability. Under the assumption that any burst may access any regenerator in a node (as shown in Section III-A, the architecture proposed assures a fair access to the regenerator pool), we make use of the inverse of the Erlang B-loss function as the dimensioning function $f$.

\section{RESULTS AND DISCUSSION}

In this Section, we present the performance results of the translucent OBS network architecture. Specifically, the RG and LCR heuristics are compared against each other and also against both the opaque and transparent cases, which are the benchmarking references in this study. Notice that, for the sake of a fair comparison, the transparent network used in this study does neither assume an ideal physical layer nor availability of optical 3R regeneration.

\section{A. Scenario}

The metric of interest in this study is the overall burst loss probability (BLP). The evaluation is accomplished through a series of simulations which consider the following simulation scenario: (1) simulations are conducted on the JAVOBS [15] network simulator; (2) the Pan-European Core (16 nodes and 23 links) and Large (37 nodes and 57 links) networks are the topologies considered [16]; (3) channel bit rate is set to $10 G b p s$; (4) the load is normalised to the link capacity; (5) bidirectional links equipped with 32 channels each; (6) the traffic is uniformly distributed; (7) the OSNR threshold is $20 d B$ (which is fairly enough taking into account the value proposed in [9]); (8) $B$ is set to $10^{-3} ;$ (9) $\left|\mathcal{P}_{d}\right|=2$.

\section{B. Results}

Figure 4 shows the results obtained considering the Core topology under four different scenarios, namely an opaque and a transparent network both operating under the MILP routing proposed by the LCR algorithm and a translucent

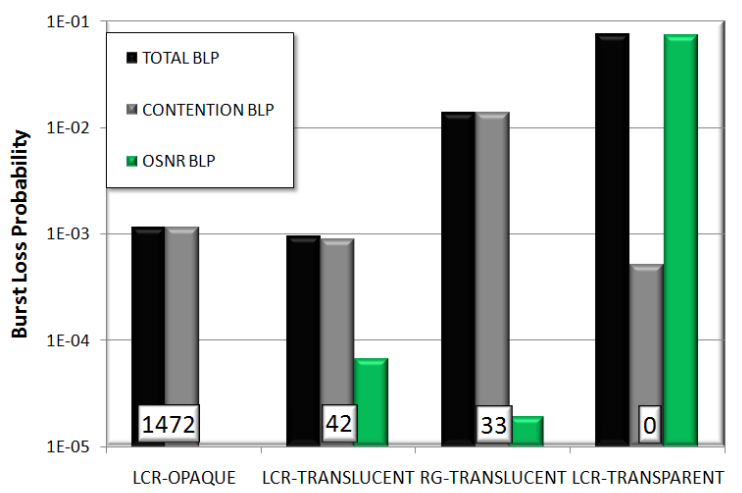

Fig. 4. Burst Loss Probability due to contention and OSNR comparing the opaque, the transparent and both the RG and LCR translucent solutions considering the Core topology.

network operating under both the LCR and RG algorithms. Besides, the total number of regenerators placed in each case is shown in the squares. The columns in the figure represent the total BLP, the BLP due to burst contention and the BLP due to bursts arrived at destination with an OSNR under the threshold, respectively. In this study, each node generates a total amount of 133 Gbps.

The result of the transparent case makes it clear that the impact of the PLIs in an OBS network is quite severe; losses are completely dominated by bursts dropped due to OSNR. On the other hand, the opaque solution provides the lowest BLP but requires a vast number of regenerators (1472). It is easy to observe that for the proposed translucent architecture, the BLP of both the LCR and RG strategies are dominated by the contention ones, which means that OSNR losses are maintained under control thanks to the regenerators placed in the network. Although the RG method requires slightly less regenerators, its routing decisions lead to a poor network performance. The LCR strategy, by contrast, attains the performance of the opaque case, but more importantly, LCR only needs $3 \%$ of the regenerators considered in the opaque solution. In further analysis on the Large topology, not provided here due to space limitations, similar results were observed. Hence, hereinafter we only consider the LCR algorithm for the evaluation of our translucent OBS network architecture.

In Fig. 5, we depict the BLP performance with respect to the number of regenerators placed in the network. We consider the Core topology and that each node generates 142Gbps. Both the opaque and transparent cases are plot and used as benchmarking indicators. As it was to be expected, the performance of the translucent topology is clearly bounded by that of both the opaque and transparent network. The LCR heuristic requires 45 regenerators to meet the OSNR requirements. Notice that, the performance of the translucent network with more than 40 regenerators is slightly better than that of the opaque one (which needs an unfeasible number of regenerators) because in the former, bursts undergoing $\mathrm{O} / \mathrm{E} / \mathrm{O}$, make use of electrical buffers at the regenerator pool and, 


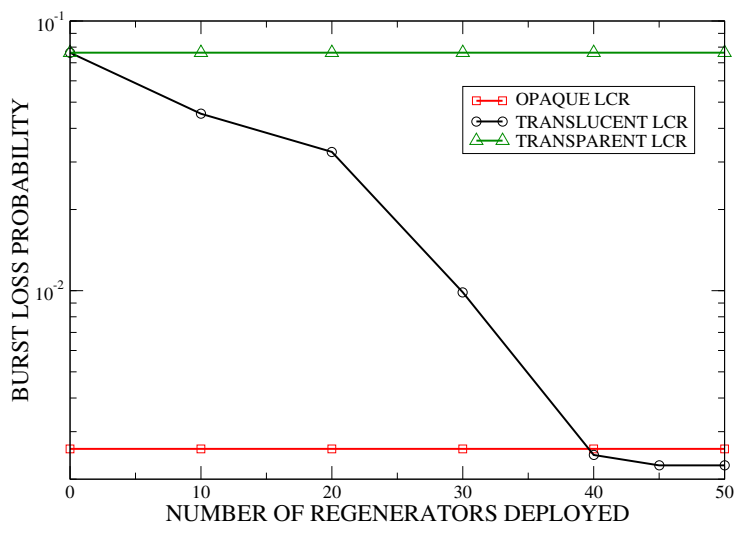

Fig. 5. Burst Loss Probability of the LCR algorithm as a function of the number of regenerators considering the Core topology.

therefore, the contention is slightly reduced.

Eventually, in Fig. 6, we assess how effective at maintaining under control the OSNR losses the LCR strategy is. In this experiment, the Large topology is considered. One can note that, whilst OSNR losses remain nearly flat regardless of the network load, contention losses become dominant as the load increases. The number of regenerators required in each case is displayed in the top x-axis. Again, OSNR losses are smoothly decreased as a consequence of the high contention losses.

\section{Vi. CONClusions AND Future WORK}

In this paper, we have focused on the problem of PLIs in OBS networks. In particular, we have proposed a novel translucent OBS network architecture consisting of all-optical TAS nodes equipped with a limited number of $\mathrm{O} / \mathrm{E} / \mathrm{O}$ regenerators. We have provided an OSNR model to evaluate the impact of the main PLIs (i.e., ASE noise and splitting losses) and illustrated a method to compute a power budget and noise analysis. This model has been then used to define two RRP heuristics, namely RG and LCR. Performance results indicate that both heuristics successfully maintain negligible bursts losses due to intolerable OSNR. Among them, LCR, which is based on a MILP routing model, attains the performance of the opaque network [8] but requiring a reduced amount of regenerators (e.g., 43 vs. 1472 , in Core topology and 528 vs. 3648 , in Large topology).

Despite both the RG and LCR techniques do not provide an optimal RRP, they have allowed to gain a valuable insight into the evaluation of our translucent OBS network architecture. It is for this reason that, in our future work, we plan to extend the present study including a formal model for the RRP problem. Another subject which certainly deserves further research is the consideration of an on-line/dynamic approach.

\section{ACKNOWLEDGMENT}

The research leading to these results has received funding from the European Community's Seventh Framework Programme FP7/2007-2013 under grant agreement no. 247674 (the STRONGEST project). The authors thank the support received from the Spanish Ministry of Science and Innovation

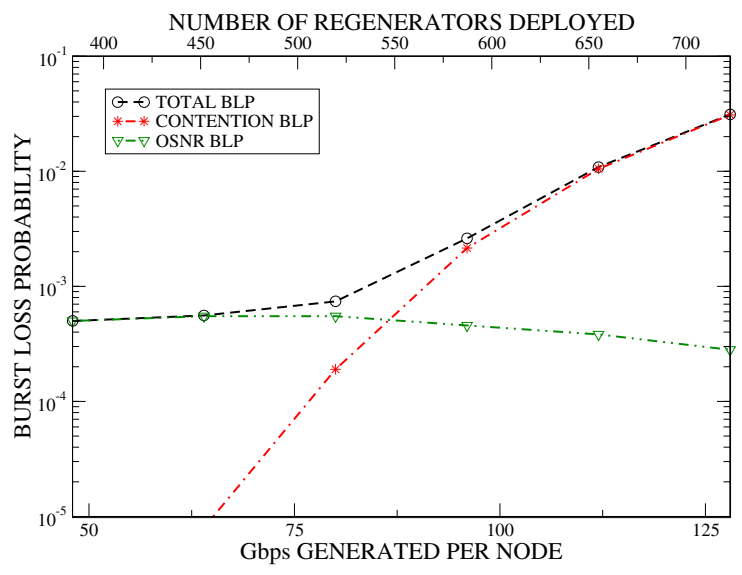

Fig. 6. Burst Loss Probability due to contention and OSNR of the LCR algorithm as a function of the offered load considering the Large topology.

(contract TEC2009-13252), the Catalan Government (contract SGR-1140), and the Polish Ministry of Science and Higher Education (contract 643/N-COST/2010/0). The authors would also like to thank Sébastien Rumley (EPFL, Switzerland) for the development of the Javanco software framework.

\section{REFERENCES}

[1] S. Azodolmolky et al., "A survey on physical layer impairments aware routing and wavelength assignment algorithms in optical networks," Com. Netw., vol. 53, no. 7, May 2009.

[2] B. Ramamurthy et al., "Transparent vs. opaque vs. translucent wavelength-routed optical networks," in Proc. OFC 1999, Feb. 1999.

[3] G. Shen and R. S. Tucker, "Translucent optical networks: the way forward," IEEE Commun. Mag., vol. 45, no. 2, Feb. 2007.

[4] A. Sen, S. Murthy, S. Bandyopadhyay, "On sparse placement of regenerator nodes in translucent optical network," in Proc. IEEE Globecom 2008, Nov. 2008.

[5] W. Zhang et al., "REPARE: Regenerator placement and routing establishment in translucent networks," in Proc. IEEE Globecom 2009, Nov. 2009.

[6] Y. Fan, B. Wang, "Physical impairment aware scheduling in optical burst switched networks," Phot. Netw. Commun., vol. 18, no. 2, Oct. 2009.

[7] B.G. Bathula, V.M. Vokkarane, R.R.C. Bikram, "Impairment-aware manycasting over optical burst-switched networks," in Proc. IEEE ICC 2008, May 2008.

[8] H. Buchta and E. Patzak, "Analysis of the physical impairments on maximum size and throughput of SOA-based optical burst switching nodes," IEEE/OSA J. Lightwave Technol., vol. 26, no. 16, Aug. 2008.

[9] H. Pereira et al., "OSNR model to consider physical layer impairments in transparent optical networks," Phot. Netw. Commun., vol. 18, no. 2, Oct. 2009

[10] R. Martínez et al., "Experimental GMPLS routing for dynamic provisioning in translucent wavelength switched optical Networks," in Proc. OFC/NFOEC 2009, San Diego, USA, Mar. 2009.

[11] T. Tsuritani et al., "Optical path computation element interworking with network management system for transparent mesh networks," in Proc. OFC/NFOEC 2008, San Diego, USA, Feb. 2008.

[12] INPHENIX: http://www.inphenix.com/soa_devices.html, accessed in Mar. 2010.

[13] MRV: http://www.mrv.com/product/MRV-LD-OAB, accessed in Mar. 2010.

[14] M. Klinkowski et al., "An overview of routing methods in optical burst switching networks," Opt. Swit. Netw., vol. 7, no. 2, Apr. 2010.

[15] O. Pedrola et al., "JAVOBS: a flexible simulator for OBS network architectures," J. Netw., vol. 5, no. 2, Feb. 2010.

[16] S. De Maesschalck et al., "Pan-European Optical Transport Networks: An Availability-based Comparison," Phot. Netw. Commun., vol. 5, no. 3 , May 2003. 